\title{
Genetic mapping of aphicarus - a sex-linked locus controlling a wing polymorphism in the pea aphid (Acyrthosiphon pisum)
}

\author{
C Braendle ${ }^{1,2}$, MC Caillaud ${ }^{3}$ and DL Stern ${ }^{2}$ \\ ${ }^{1}$ Laboratory for Development and Evolution, University Museum of Zoology, Department of Zoology, University of Cambridge, \\ Cambridge CB2 3EJ, UK; ${ }^{2}$ Department of Ecology E Evolutionary Biology, Princeton University, Princeton, NJ 08544, USA; ${ }^{3}$ Biology \\ Department, Ithaca College, Ithaca, NY 14850, USA
}

\begin{abstract}
We have initiated research to determine the genetic basis of a male wing polymorphism in the pea aphid Acyrthosiphon pisum (Hemiptera: Aphididae). Previous studies showed that this polymorphism is controlled by a single biallelic locus, which we name aphicarus (api), on the $\mathrm{X}$ chromosome. Our objectives were to confirm that api segregates as a polymorphism of a single gene on the $\mathrm{X}$ chromosome, and to obtain molecular markers flanking api that can be used as a starting point for high-resolution genetic and physical mapping of the target region, which will ultimately allow the cloning of api. We have established an $F_{2}$ population segregating for api and have generated X-linked AFLP markers. The segregation pattern of api in the $\mathrm{F}_{2}$ population
\end{abstract}

shows that the male wing polymorphism segregates as a polymorphism of a single gene, or set of closely linked genes on the $X$ chromosome. Using a subset of $78 \mathrm{~F}_{2}$ males, we have constructed a linkage map of the chromosomal region encompassing api using seven AFLP markers. The map spans $74.1 \mathrm{cM}$ and we have mapped api to an interval of $10 \mathrm{cM}$. In addition, we confirmed $X$ linkage of our AFLP markers and api by using one $X$-linked marker developed in an earlier study. Our study presents the first mapping of a gene with known function in aphids, and the results indicate that target gene mapping in aphids is feasible.

Heredity (2005) 94, 435-442. doi:10.1038/sj.hdy.6800633 Published online 26 January 2005

Keywords: AFLP; alternative phenotypes; aphicarus (api); wing polymorphism; wing polyphenism

\section{Introduction}

Many organisms have the capacity to switch between developmental pathways that result in distinctly different phenotypes. The primary factors regulating the expression of such alternative phenotypes may be exclusively genetic (polymorphism) or their action may further depend on environmental signals (often called polyphenism). Alternative phenotypes are usually specialized for different tasks or habitats and exhibit corresponding differences in physiology, morphology and behaviour (Moran, 1992; Roff, 1996). The production of alternative phenotypes enables an organism to conduct alternative life styles by exploiting different resources, which may allow a division of labour among phenotypes specialized for different tasks. The production of alternative phenotypes is thus considered to be adaptive in many instances. Moreover, alternative phenotypes may play significant roles in the evolution of novelty and rates of speciation (West-Eberhard, 1989). However, despite the widely recognized importance of alternative phenotypes in many biological phenomena, the genetic basis underlying such discrete phenotypic variation has rarely been elucidated.

Correspondence: C Braendle. Current address: Institut Jacques Monod, CNRS, Universités Paris 6 and 7, Tour 43, 2 place Jussieu, 75251 Paris cedex 05, France. E-mail: braendle@ijm.jussieu.fr

Received 5 March 2004; accepted 26 October 2004; published online 26 January 2005
Wing polymorphism and polyphenism of insects represent well-studied examples of alternative phenotypes (Harrison, 1980; Roff, 1986; Zera and Denno, 1997). Insects across a wide range of taxa have evolved the capacity to develop flight-capable (fully winged) phenotypes or flight-incapable (wingless or short-winged) phenotypes. The winged phenotype is generally responsible for long-range dispersal and exhibits a lower fecundity than the wingless, sedentary phenotype. This trade-off between flight capability and fecundity appears to be the driving force maintaining intraspecific variation in wing morphology (Zera and Denno, 1997). In some species, the alternative adopted during development may depend on the alleles present at a single locus. More commonly, the production of alternative phenotypes appears to be under polygenic control and to be condition-dependent, that is, specific environmental conditions induce the developing animal to adopt one of the alternatives (Roff and Fairbairn, 1991). (Note that both wing polymorphism and wing polyphenism are often referred to in the literature as wing polymorphism.)

From a physiological perspective, wing polyphenism/ polymorphism is now one of the best-understood life history trade-off models (Zera and Harshman, 2001). In contrast, we have limited information on the genetic mechanisms controlling the production of such alternative phenotypes. Most importantly, we do not know which genes play a direct role in causing divergence of alternative developmental pathways. In general, it is difficult to identify such critical genes in a polyphenic 
switch because most currently used assays, such as differential gene expression analysis, generate candidate genes that are likely to result from phenotype-specific expression after developmental divergence. Nevertheless, recent studies using this approach have provided the first genetic insights into polyphenic development (eg Evans and Wheeler, 1999; Miura et al, 1999). Similar problems arise when adopting a candidate gene approach: as alternative phenotypes generally differ in a suite of morphological characters and because the time point of developmental divergence is often known only at the phenotypic level, the selection of potential candidate genes to be analysed is difficult. Hence, it is more feasible to identify genes critical for the regulation of alternative phenotypes where the underlying genetic determinants can be mapped using classic genetic analysis. To our knowledge, however, no studies have so far attempted to map any genes involved in the regulation of insect wing polymorphisms.

The pea aphid Acyrthosiphon pisum (Hemiptera: Aphididae), like most aphid species, exhibits a life cycle involving several parthenogenetic generations alternating with a single sexual generation (cyclical parthenogenesis). During the parthenogenetic mode of reproduction, females display a wing polyphenism. Environmental cues, such as crowding or low host plant quality, trigger the production of winged females that disperse and colonize new host plants (eg Lees, 1966). In the sexual generation, females are always wingless, while males are either winged or wingless. This phenotypic difference among pea aphid males is under exclusively genetic control, thus representing a wing polymorphism (Smith and MacKay, 1989; Caillaud et al, 2002).

Initially, Smith and MacKay (1989) reported the occurrence of three types of pea aphid clones collected in nature: clones producing only winged males, clones producing only wingless males and clones producing an equal proportion of winged and wingless males. As aphid sex determination is of the XX/X0 (female/male) type (Blackman, 1987), Smith and MacKay (1989) proposed that the male wing polymorphism segregates as an X-linked factor, most likely as a single locus with two alternative alleles. Males produced by females homozygous at the locus would therefore produce either only winged or only wingless males. In contrast, heterozygous females would produce an equal proportion of winged and wingless males. Caillaud et al (2002) tested the genetic model proposed by Smith and MacKay (1989) by analysing the segregation pattern of the male wing polymorphism in $F_{1}$ and $F_{2}$ generations derived from parental clones producing either winged or wingless males only (ie clones presumptively homozygous for the alternative alleles at the locus). The segregation pattern of the polymorphism was consistent with $X$ linkage and a biallelic single locus control. The $X$ linkage of the locus was further demonstrated by the linkage of X-linked microsatellite markers with the phenotypic polymorphism in heterozygous $\mathrm{F}_{1}$ clones.

The male wing polymorphism in the pea aphid provides a relatively simple, suitable system to dissect the molecular genetic basis underlying the expression of alternative phenotypes. We have named this gene aphicarus, abbreviated api (after the tragic figure from Greek mythology, Icarus, whose wax and feather wings melted when he flew too close to the sun). In this study, we generated amplified fragment length polymorphism (AFLP) markers for the pea aphid $X$ chromosome using an $\mathrm{F}_{2}$ mapping population segregating for the male wing polymorphism. Our major goals of this mapping project were to confirm $X$ linkage of api, to determine whether api segregates as a polymorphism of a single gene, and to obtain molecular markers flanking api that can be used as a starting point for high-resolution genetic and physical mapping of the target region, which will ultimately allow the cloning of api.

\section{Materials and methods}

\section{Phenotype of winged and wingless males}

In the pea aphid, the winged male is fully winged and has functional flight muscles. In comparison to the wingless male, the winged phenotype exhibits heavier sclerotization of head and thorax, better-developed compound eye ocelli, longer antennae with more rhinaria, and the siphunculi and cauda may sometimes be longer (eg Kring, 1977; Kawada, 1987; Miyazaki, 1987). Furthermore, alternative phenotypes may differ in body length and body weight, with the winged phenotype usually being smaller. The morphological differences between winged and wingless phenotypes correlate with differences in life history traits. For example, the winged male shows an increased postembryonic developmental time due to a prolonged fourth larval instar.

\section{Terminology}

We use the abbreviations $a p i^{w}$ and $a p i^{w l}$ to refer to the alleles of aphicarus causing winged and wingless males, respectively. Note that it is not possible to determine whether there is a dominance relationship between these alleles, since males are haploid for the X chromosome (and therefore aphicarus) and this phenotype is expressed only in males. In a previous study, the api locus was referred to as 'WgMa' locus (Caillaud et al, 2002).

\section{Genotyping at the api locus}

The api genotype for all aphid clones was determined by inducing the production of males in parthenogenetic clones (Caillaud et al, 2002). We induced the production of sexual morphs by transferring 3-4 third-instar nymphs from long-day conditions (L:D 16:8, 20 ${ }^{\circ} \mathrm{C}$ ) to short-day conditions (L:D 13:11, $17^{\circ} \mathrm{C}$ ). Sexual morphs started appearing approximately 8 weeks after transfer. Clones that produced only wingless males were typed as $a p i^{w l} / a p i^{w l}$, clones that produced only winged males were typed as $a p i^{w} / a p i^{w}$ and clones that produced both male morphs were typed as $a p i^{w} / a p i^{w l}$. Clones producing at least five males all of a single morph were considered to be homozygous for the corresponding api allele.

\section{$\mathrm{F}_{2}$ mapping population}

Two parental clones, PBR8 and LSR1, were used to generate the $\mathrm{F}_{2}$ mapping population. PBR8 is homozygous for apiw and LSR1 is homozygous for apiwl (Caillaud et al, 2002). The parental clones originated from the same population and were collected in the summer of 1998 in alfalfa fields (Medicago sativa) in Tompkins county, NY, USA. Parental, $F_{1}$ and $F_{2}$ clones 
were maintained in the laboratory on $M$. sativa or Medicago arborea at $20^{\circ} \mathrm{C}$, L:D $16: 8$ and cultured as previously described (Caillaud et al, 2002; Miura et al, 2003).

Crosses were performed as described by Via (1992) and Caillaud et al (2002). Briefly, virgin females isolated as nymphs from the sexual stock cultures were mated to males in Petri dishes. All fertilized eggs produced throughout the life of the females were harvested, surface sterilized and placed in an incubator under daily cycles of $4{ }^{\circ} \mathrm{C}$ during a $10 \mathrm{~h}$ day and $0^{\circ} \mathrm{C}$ during a $14 \mathrm{~h}$ night. After 100 days of this cold treatment, eggs were removed from the incubator and the hatchling progeny were reared in Petri dishes containing alfalfa foliage. The hatching rates for eggs resulting from the different crosses (see below) were relatively high for aphids (cf Via, 1992), ranging from 82 to $87 \%$.

Figure 1 depicts the crosses performed. $F_{1}$ hybrids were obtained from reciprocal crosses between sexuals of parental clones PBR8 and LSR1: female PBR8 $\times$ male LSR1 $\left(\mathrm{F}_{1-\mathrm{P} \times \mathrm{L}}\right.$ hybrids $)$ and female LSR1 $\times$ male PBR8 ( $F_{1-L \times P}$ hybrids). To produce the $F_{2}$ generation, we randomly selected one $\mathrm{F}_{1-\mathrm{P} \times \mathrm{L}}$ and one $\mathrm{F}_{1-\mathrm{L} \times \mathrm{P}}$ hybrid and reciprocally crossed them (Figure 1):

$\mathrm{F}_{2}$ cross 1: $\mathrm{F}_{1-\mathrm{P} \times \mathrm{L}}$ api $i^{w} / a p i^{w l}$ female $\times \mathrm{F}_{1-\mathrm{L} \times \mathrm{P}}$ api $i^{w}$ male $\mathrm{F}_{2}$ cross $2: \mathrm{F}_{1-\mathrm{P} \times \mathrm{L}}$ apiw $/$ api $i^{w l}$ female $\times \mathrm{F}_{1-\mathrm{L} \times \mathrm{P}}$ api ${ }^{w l}$ male

$\mathrm{F}_{2}$ cross 3: $\mathrm{F}_{1-\mathrm{L} \times \mathrm{P}}$ api ${ }^{w} /$ api $i^{w l}$ female $\times \mathrm{F}_{1-\mathrm{P} \times \mathrm{L}}$ apiw male $\mathrm{F}_{2}$ cross 4: $\mathrm{F}_{1-\mathrm{L} \times \mathrm{P}}$ apiw $/$ api $i^{w l}$ female $\times \mathrm{F}_{1-\mathrm{P} \times \mathrm{L}}$ apiwl male

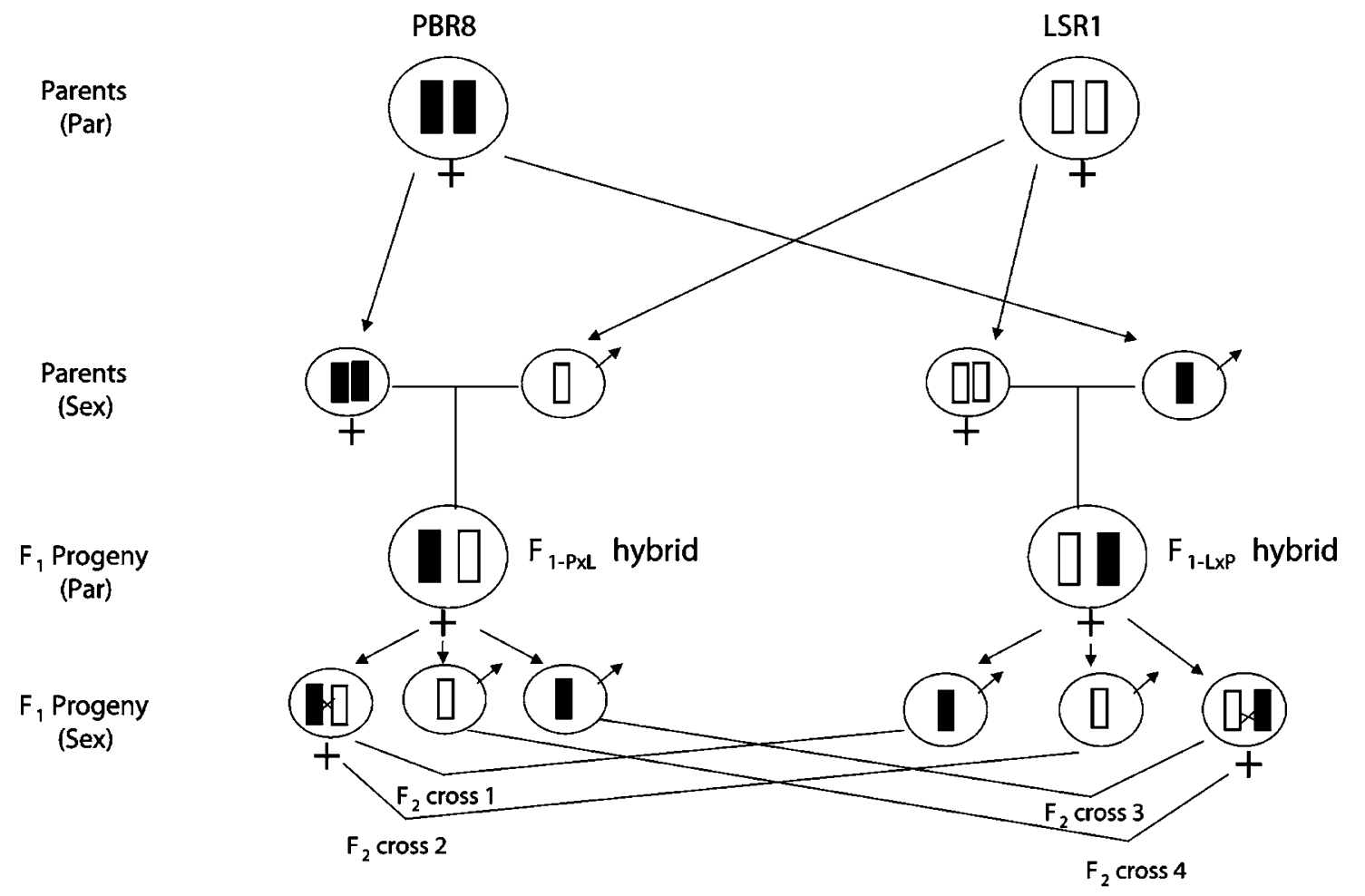

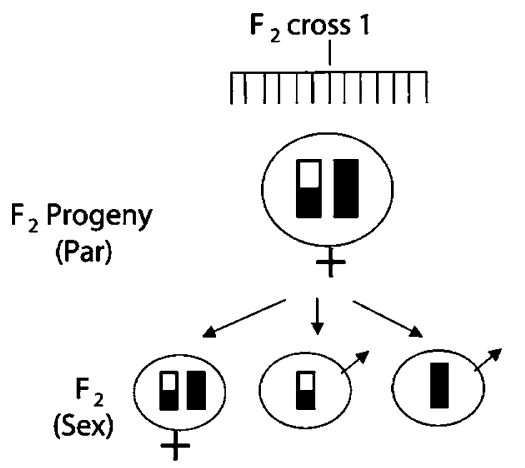

(a)

(b)

(c)
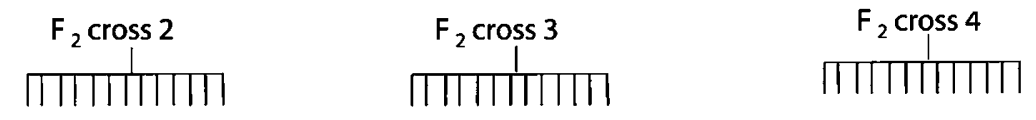

Figure 1 Schematic illustration of $\mathrm{F}_{2}$ population segregating for api and selection of informative $\mathrm{F}_{2}$ males for linkage analysis. The black and white bars represent the respective parental $\mathrm{X}$ chromosomes with different api alleles. Parental females of PBR8 are homozygous for api ${ }^{w}$ (black chromosome). Parental females of LSR1 are homozygous for api ${ }^{\text {wl }}$ (white chromosome). The parthenogenetic (par) females (large circles) are diploid for the $\mathrm{X}$ chromosome. The sexual (sex) generation (small circles) consists of sexual females, which are diploid for the $\mathrm{X}$ chromosome, and males, which are haploid for the $X$ chromosome. The sexual $F_{2}$ generation consists of diploid females (a, $\left.d, g, j\right)$ and haploid males $(b, c, e, f, h, i, k, l) . F_{2}$ males $c, f, h$ and $k$ inherited the nonrecombinant $X$ chromosome from their $F_{1}$ father. In contrast, $F_{2}$ males $b, e, i$ and $\mathrm{l}$ inherited an $\mathrm{X}$ chromosome containing informative recombination events. 
A total of approximately $500 \quad \mathrm{~F}_{2}$ hybrids were generated, and we determined the api genotype of a random sample of $170 \mathrm{~F}_{2}$ hybrids. However, of these clones, only a subset of $\mathrm{F}_{2}$ males was used for the following reasons. First, we screened $\mathrm{F}_{2}$ males rather than $F_{2}$ females because males are haploid for the $X$ chromosomes. For X-linked markers, we were thus able to avoid the problem of dominance in scoring AFLPs. Second, we only used $F_{2}$ males that inherited the $X$ chromosome from their $\mathrm{F}_{1}$ mother. As paternally inherited $\mathrm{X}$ chromosomes are nonrecombinant due to male haploidy of the $X$ chromosome, only $F_{2}$ males with a potentially recombinant $X$ chromosome from their $F_{1}$ mother are informative for meiotic mapping. In Figure 1, $\mathrm{F}_{2}$ males $\mathrm{c}, \mathrm{f}, \mathrm{h}$ and $\mathrm{k}$ inherited the nonrecombinant $\mathrm{X}$ chromosome of their $F_{1}$ father, thus males of this type were not used for mapping. In contrast, $\mathrm{F}_{2}$ males $\mathrm{b}, \mathrm{e}, \mathrm{i}$ and 1 all harbour a maternally inherited $\mathrm{X}$ chromosome potentially containing informative recombination events. Consequently, in $\mathrm{F}_{2}$ clones homozygous at api, the parental origin of the $X$ chromosome in males could not be determined and these males were excluded from further analysis $(N=89)$. Of the remaining informative $\mathrm{F}_{2}$ hybrids (ie clones heterozygous at api, $N=81$ ), $78 \mathrm{~F}_{2}$ male descendants could be used for marker analysis: wingless males $\left(a i^{i w l}\right)$ from two crosses $\left(F_{2}\right.$ cross 1: $N=5$; $\mathrm{F}_{2}$ cross 3: $\left.N=48\right)$ and winged males $\left(a p i^{w}\right)$ from two crosses $\left(\mathrm{F}_{2}\right.$ cross $2: N=19 ; \mathrm{F}_{2}$ cross $\left.4: N=6\right)$. Note that differences in the number of $\mathrm{F}_{2}$ progeny between crosses arose from differential production of sexual forms that were used to generate the $F_{2}$ population (the differences therefore do not reflect differences in viability).

\section{DNA extraction}

Aphids used for AFLP analysis were stored in acetone (Fukatsu, 1999) at $-20^{\circ} \mathrm{C}$ prior to DNA extraction. From each $\mathrm{F}_{2}$ clone, DNA was extracted from 1-5 adult males using the Qiagen DNeasy Tissue kit (QIAGEN, Chatsworth, CA, USA). DNA was eluted in 20-100 $\mu \mathrm{l} \mathrm{TE} \mathrm{buffer}$ (20 $\mu \mathrm{l}$ per individual). The concentration of the eluted DNA was estimated by running a fraction of the sample on a $2 \%$ agarose gel.

\section{AFLP assays}

AFLP assays were performed as described previously (Vos et al, 1995), using the AFLP Analysis System II (AFLP Small Genome Primer Kit) (GIBCO BRL). AFLP fragments were generated according to the manufacturer's protocol with minor modifications as outlined below. For each reaction, an equal amount of DNA, that is, 3-9 $\mu \mathrm{l}$, of the eluted DNA was digested using EcoRI (6$\mathrm{bp}$ recognition site) and MseI (4-bp recognition site) restriction endonucleases in a reaction volume of $12.5 \mu \mathrm{l}$. Following ligation of adaptors, $2.5 \mu \mathrm{l}$ of this template was amplified in a $25 \mu \mathrm{l}$ volume using a primer pair based on the sequences of the EcoRI and MseI adapters with one selective nucleotide at the $3^{\prime}$ end. The PCR products of the preselective amplification were diluted 25 -fold, and $2.5 \mu \mathrm{l}$ of this stock was used for selective amplifications. The primers used for selective amplifications had two and three additional selective nucleotides (EcoRI +2 primers and $\mathrm{MseI}+3$ primers). Prior to selective amplification, EcoRI primers were end-labelled with ${ }^{33} \mathrm{P}$. PCR reactions were carried out in a reaction volume of $10 \mu \mathrm{l}$ over 13 cycles with a denaturation step for $30 \mathrm{~s}$ at $94^{\circ} \mathrm{C}$, an annealing step for $30 \mathrm{~s}$ at $65^{\circ} \mathrm{C}$ with a temperature decrease of $0.7^{\circ} \mathrm{C}$ per cycle, and an extension step of $1 \mathrm{~min}$ at $72^{\circ} \mathrm{C}$, followed by 30 cycles with a denaturation step for $30 \mathrm{~s}$ at $94^{\circ} \mathrm{C}$, an annealing step for $30 \mathrm{~s}$ at $56^{\circ} \mathrm{C}$, and an extension step for $1 \mathrm{~min}$ at $72^{\circ} \mathrm{C}$. All PCR amplifications were performed using a Techne Genius thermocycler.

\section{Gel analysis}

Prior to gel electrophoresis, reaction products of the selective amplification were mixed with an equal volume $(10 \mu \mathrm{l})$ of formamide dye (98\% formamide, $10 \mathrm{mM}$ EDTA, $0.05 \%$ bromophenol blue, $0.05 \%$ xylene cyanol) and denatured at $90^{\circ} \mathrm{C}$ for $3 \mathrm{~min}$. After preheating an $8 \%$ LongRanger polyacrylamide gel (BioWhittaker Molecular Applications) containing $7 \mathrm{M}$ urea and $1 \times \mathrm{TBE}$ for $30-60 \mathrm{~min}, 3 \mu \mathrm{l}$ of each sample was loaded onto the gel and run for $2 \mathrm{~h}$ at $70-80 \mathrm{~W}$ in $1 \times$ TBE buffer (at a temperature of $40-50^{\circ} \mathrm{C}$ ). After electrophoresis, gels were dried on a gel drier (BioRad) and exposed to Biomax MS Kodak Film for 2-6 days.

AFLP reactions for each primer pair combination were run on a single gel and included the male $\mathrm{F}_{2}$ progeny $(N=78)$, parental females $(N=2)$ and $F_{1}$ females $(N=2)$. We visually scored AFLP fragments as present or absent and excluded ambiguous or missing samples. To ensure accurate scoring, we scored all polymorphic bands twice, and presumptive $X$-linked polymorphisms were scored a third time. Markers were named according to the primer pair combinations used in the selective amplification step: 'EXX-MYYYN', where ' $E$ ' refers to the EcoRI primer with two selective nucleotides ' $\mathrm{XX}$ ', ' $\mathrm{M}$ ' refers to the MseI primer with the selective nucleotides ' $Y Y Y^{\prime}$ and $\mathrm{N}$ is the marker number for that primer pair combination.

\section{Linkage analysis and mapping of the api locus}

We used the following protocol to select presumptive $X$-linked markers. Males are haploid for the X chromosome, and the segregation pattern of X-linked markers in an $F_{2}$ intercross is identical to the segregation pattern in a backcross. Thus, $\mathrm{X}$-linked AFLP markers in male $\mathrm{F}_{2}$ progeny are expected to segregate in a 1:1 ratio and autosomal AFLP markers that are inherited in a dominant fashion in a 3:1 ratio. Note here that we have no reason to suspect segregation distortion for these markers because we did not find evidence of segregation distortion at the api locus (since induced parthenogenetic females always produce winged and wingless males in a 1:1 ratio) (Caillaud et al, 2002; this study). We performed $\chi^{2}$-tests for all AFLP polymorphisms to determine whether the segregation ratios of band presence/absence in the $F_{2}$ populations were significantly different from the expected 1:1 and 3:1 ratios. Significance levels were corrected for multiple comparisons $(\alpha / N)$ to give an experimentwise $\alpha=0.05$.

We performed maximum-likelihood linkage analysis using MAPMAKER/Exp Version 3.0b (Lander et al, 1987) to calculate marker order and position of the target locus on the $\mathrm{X}$ chromosome. Map distances in centimorgans (cM) were derived from recombination frequencies using the Kosambi mapping function (Kosambi, 1944). The marker data were analysed according to Mapmaker's $F_{2}$ backcross $(\mathrm{A}-\mathrm{H})$ scheme, which correctly estimates the 
recombination fractions and LOD scores for loci in haploid individuals. Marker alleles of each male were scored as ' $A$ ' (inherited from parent LSR1) or ' $B$ ' (inherited from parent PBR8), and missing or ambiguous samples were scored as ' $\mathrm{M}^{\prime}$ (missing). We confirmed linkage of selected AFLP markers to a single linkage group at a LOD score of $\geq 4.0$ and a maximum recombination fraction of $0.33(40 \mathrm{cM})$ using the 'group' command. Map order of AFLP markers and position of the target locus were determined using the 'compare' and 'try' commands. Marker orders were verified using the 'ripple' command. MAPMAKER's 'error detection' command was used to identify candidate genotyping errors (Lincoln and Lander, 1992).The resulting linkage map was plotted using the software program Mapchart 2.1 (Voorrips, 2002).

We also determined the number of presumptive autosomal linkage groups using Mapmaker's $\mathrm{F}_{2}$ intercross (A-B-C-D) scheme. For markers segregating 3:1 (banded: unbanded) among the male $F_{2}$ progeny, band absence was coded as A for homozygous for the parental allele a and band presence as $C$ for nonhomozygous for parental allele $\mathrm{a}$, when the band was absent in parent $\mathrm{A}$ (LSR1). Band absence and presence in parent $B$ were coded correspondingly as B and D for parent B (PBR8).

\section{Analysis of previously identified X-linked markers}

Hawthorne and Via (2001) constructed an AFLP linkage map of the pea aphid and cloned two markers $(\mathrm{SX} 380=$ Codom380, Pag-Egc277 $=$ Codom277) on the putative $X$ chromosome (D Hawthorne, personal communication). We screened the two markers for size polymorphisms between the parental strains. SX380 showed no length polymorphisms. Marker Pag-Egc277 was polymorphic in one parent (LSR1) and monomorphic in the other (PBR8). Only one of the $F_{1}$ clones $\left(\mathrm{F}_{1-\mathrm{P} \times \mathrm{L}}\right)$ was polymorphic for Pag-Egc277 and we scored the male $F_{2}$ progeny that inherited their $X$ chromosome from this $\mathrm{F}_{1}$ clone $(N=24)$ for their genotype at this locus and used the resulting data for linkage analysis. Marker amplifications were carried out in $15 \mu$ l reaction mixtures consisting of $0.2 \mathrm{U}$ of Taq polymerase, $1 \times$ reaction buffer with $\mathrm{MgCl}_{2}, 200 \mu \mathrm{M}$ dNTPs, $0.2 \mathrm{mM}$ of each primer (5'-CAATATGCCTGTGAGGTTGG-3', 5'-GTGAATTTTA CAAGTTTTTGCTAG-3') and $1 \mu \mathrm{l}$ of DNA ( $5 \mathrm{ng} / \mu \mathrm{l})$. PCR reactions were amplified using a regime of initial denaturation at $94^{\circ} \mathrm{C}$ for $3 \mathrm{~min}$, followed by 40 cycles of denaturation at $94^{\circ} \mathrm{C}$ for $1 \mathrm{~min}$, annealing at $45^{\circ} \mathrm{C}$ for $1 \mathrm{~min}$ and elongation at $72^{\circ} \mathrm{C}$ for $45 \mathrm{~s}$. This was followed by a final extension step at $72^{\circ} \mathrm{C}$ for $4 \mathrm{~min}$.

\section{Results}

The segregation of api in the $\mathrm{F}_{2}$ generation was consistent with the previously proposed $X$ linkage of api (Smith and MacKay, 1989; Caillaud et al, 2002). $\mathrm{F}_{2}$ clones produced either both types of males, only winged males or only wingless males, and the ratio of different $F_{2}$ clones derived from each cross was 1:1 (Table 1). Heterozygous $\mathrm{F}_{2}$ clones $(N=78)$ produced winged and wingless males in equal proportions (Appendix A1, Table A1).

We screened AFLP fragments amplified by 38 different primer pair combinations $(E c o R I+2 / M s e I+3)$. On average, an AFLP reaction resulted in $44.2 \pm 1.8$ (mean \pm SE) bands (range: $N=26-74$ ). The size of amplified frag-
Table 1 Pattern of male morph production in $\mathrm{F}_{2}$ clones $(N=170)$ generated by the four $\mathrm{F}_{2}$ crosses $^{\mathrm{a}}$

\begin{tabular}{lccc}
\hline$F_{2}$ cross & $\begin{array}{c}\text { Clones producing } \\
\text { winged males } \\
\left(\mathrm{api}^{\mathrm{w}} / \mathrm{api}^{\mathrm{w}}\right)\end{array}$ & $\begin{array}{c}\text { Clones producing } \\
\text { wingless males } \\
\left(\mathrm{api}^{\mathrm{wl}} / \mathrm{api}^{\mathrm{wl}}\right)\end{array}$ & $\begin{array}{c}\text { Clones producing } \\
\text { both male morphs } \\
\left(\mathrm{api}^{\mathrm{w}} / \mathrm{api}^{\mathrm{wl}}\right)\end{array}$ \\
\hline 1 & 3 & 0 & 5 \\
2 & 0 & 17 & 20 \\
3 & 54 & 0 & 50 \\
4 & 0 & 15 & 6 \\
\hline
\end{tabular}

${ }^{a}$ Each cross between the $F_{1}$ parents resulted in equal proportions of $\mathrm{F}_{2}$ clones with different api genotypes (homozygous vs homozygous) ( $\chi^{2}$-test, all $\left.P>0.05\right)$

ments ranged from approximately 70 to $1500 \mathrm{bp}$, with most bands ranging from 100 to $500 \mathrm{bp}$. Of a total of 1681 amplified fragments, $38(2.3 \%)$ were polymorphic between parental females, monomorphic (banded) in the $F_{1}$ females and segregating among the $F_{2}$ males. Segregation ratio analyses resulted in the identification of nine putative X-linked markers (1:1) and 27 putative autosomal markers (3:1). Two markers showed significant deviation from the expected ratios and were excluded from analysis.

The nine X-linked AFLP markers grouped into a single linkage group at a LOD threshold of 4.0. A subset of seven AFLP markers could be ordered unambiguously (LOD $\geq 2.0)$ using the 'compare' command. The target locus api was placed on this map at a LOD (linkage) score of 21.33 and its map position was supported by a LOD score of 2.70 ('try' command). The final map consists of seven AFLP markers plus the target locus and spans a chromosomal region of $74.1 \mathrm{cM}$ (Figure 2 ). We verified the map order of the seven AFLP markers plus the target locus using the 'compare' and 'ripple' commands. All marker positions were supported by a LOD $\geq 2.0$. The remaining two AFLP markers and marker Pag-Egc277 did not have unique map positions (LOD <2.0) (see Figure 2 for details).

Presumptive autosomal markers segregating in a 3:1 ratio in the male $F_{2}$ population grouped into three (parent PBR8, $N=14$ ) and two linkage groups (parent $\mathrm{B}$, $N=13$ ). For parent PBR8, the three linkage groups comprised nine, three and two markers (one marker was unlinked). For parent LSR1, the two linkage groups comprised four and three markers (six markers were unlinked).

\section{Discussion}

The segregation pattern of api in the $\mathrm{F}_{2}$ population supports the previously proposed model that this trait is controlled by a single X-linked locus (aphicarus) with two alternative alleles causing the winged and wingless male phenotype (Smith and MacKay, 1989; Caillaud et al, 2002). Using a subset of $78 \mathrm{~F}_{2}$ males, we generated nine X-linked AFLP markers, seven of which were used to determine the map position of api. We identified two AFLP markers confining api to a map interval of $10 \mathrm{cM}$. One marker maps $1.5 \mathrm{cM}$ from api and another marker maps $8.4 \mathrm{cM}$ on the other side of the locus. The fact that we did not find any AFLP markers that segregated perfectly with api suggests that the gene is not positioned 
440

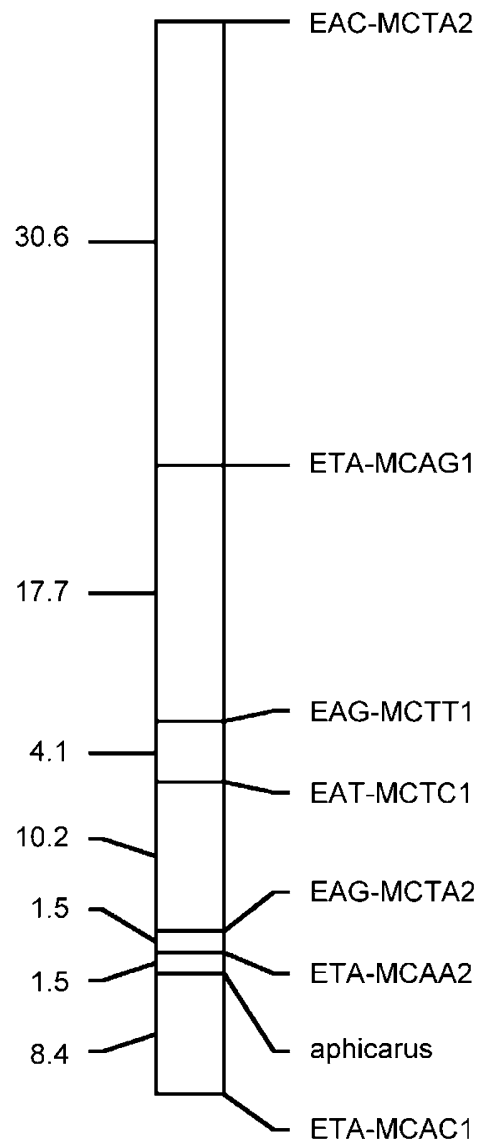

Figure 2 Genetic map of the $X$ chromosome region containing aphicarus. The linkage map consists of seven AFLP markers plus the target locus, covering $74.1 \mathrm{cM}$ with a mean intermarker distance of $10.6 \pm 3.67 \mathrm{cM}$. The map interval containing api spans $9.9 \mathrm{cM}$, delimited by markers ETA-MCAC1 $(8.4 \mathrm{cM})$ and ETA-MCAA2 $(1.5 \mathrm{cM})$. For two X-linked AFLP markers, no unique map position could be determined: ETC-MCAC1 with a LOD (linkage) score of 17.91 falls into marker interval EAG-MCTT1-ETA-MCAG1 $(\mathrm{LOD}=0.00)$ or EAG-MCTT1-EAT-MCTC1 $(\mathrm{LOD}=0.15)$ (the next best position of the marker is less likely at a LOD score of 1.86). Marker EAT-MCTC with a LOD (linkage) score of 6.19 falls into marker interval EAG-MCTA2-EAT-MCTC1 $(\mathrm{LOD}=0.00)$ or EAGMCTT1-ETA-MCAG1 (LOD =0.27) (the next best position of the marker is less likely at a LOD score of 1.44). The previously identified X-linked marker Pag-Egc277 (Hawthorne and Via, 2001) with a LOD (linkage) score of 7.22 falls in the marker interval ETAMCAA2-EAG-MCTA2 (LOD $=0.00)$ or EAG-MCTA2-EAT-MCTC1 $(\mathrm{LOD}=0.00)$, with the next best map position being less likely at a LOD score of 2.79 .

within a large inversion and that we should be able to localize it further with standard meiotic mapping.

In our AFLP assay, we found that approximately $2 \%$ of amplified fragments were polymorphic between parents and suitable for marker analysis, which corresponds to about one marker per primer pair combination. This marker recovery rate is low compared to AFLP studies on other insects using divergent parental lines (eg Hawthorne, 2001; Parsons and Shaw, 2002). However, in our study, the parental clones were derived from the same population (and the same species of host plant). In addition, genetic differentiation (inferred from mtDNA and allozyme analysis) between pea aphid clones may be low generally, even when clones are specialized for different host plants (Simon et al, 1982; Dalmasso and Bournoville, 1983; Boulding, 1998; Birkle and Douglas, 1999). Despite little genetic divergence between the parental clones used in our experiment, we were able to gain sufficient information to place the target locus within an interval of $10 \mathrm{cM}$. Our mapping experiment was facilitated by the fact that aphid males are haploid for the $X$ chromosome, resulting in complete haplotype information for X-linked AFLP markers. In addition, using api as a marker, we were able to select an $\mathrm{F}_{2}$ mapping population of informative males and avoided screening males with paternally derived, nonrecombinant $X$ chromosomes.

The pea aphid genome has a haploid set of four chromosomes (Sun and Robinson, 1966). In addition to the linkage group corresponding to the $X$ chromosome, we found that presumptive autosomal markers grouped into three and two linkage groups for the parents PBR8 and LSR1. The recovery of only two autosomal linkage groups for parent LSR1 may have resulted from the small number of markers available.

Hawthorne and Via (2001) generated a whole-genome AFLP linkage map of $A$. pisum using a female $\mathrm{F}_{2}$ population. The two parental maps corresponding to the $X$ chromosomes were approximately $30 \mathrm{cM}$ (five markers) and $100 \mathrm{cM}$ (12 markers). In this study, we used seven AFLP markers (plus the target locus) to construct a map that covered a distance of $74 \mathrm{cM}$. Therefore, it seems likely that these differences in map length stem from differences in the numbers of markers used to generate these maps. Due to the small number of markers, this region is unlikely to span the entire $X$ chromosome. The map length of $100 \mathrm{cM}$ by Hawthorne and Via (2001) may be the most reliable estimate of the genetic length of the pea aphid X chromosome as this estimate is based on the largest number of markers.

We were unable to unambiguously map the X-linked marker Pag-Egc277 developed in the study of Hawthorne and Via (2001), but this marker grouped with our $X$ chromosome linkage group at a high LOD score. Pag-Egc277 appears to map somewhere between markers ETA-MCAA2 and EAT-MCTC1, suggesting that it is located within 1.5-13.2 cM of api (Figure 2). Although we cannot unambiguously orient our map with the map of Hawthorne and Via (2001) based on this single marker, the relatively telomeric location of Pag-Egc277 (approximately $10 \mathrm{cM}$ from the end of their map and 9.9-21.6 from the end of our map) suggests that our terminal marker ETA-MCAC1 is located on the same chromosome end as their terminal marker E2-600 and that api is located within approximately $10 \mathrm{cM}$ of the end of both maps.

We currently estimate the pea aphid X chromosome to cover approximately $30 \%$ of the genome, based on several chromosome photographs (Mandrioli et al, 1999; Bizzaro et al, 2000, RL Blackman, unpublished data). The pea aphid genome is relatively small and estimated to be $300 \mathrm{Mb}$ (Finston et al, 1995). The $\mathrm{X}$ chromosome thus contains approximately $90 \mathrm{Mb}$ and the estimated relationship between physical and genetic distance is approximately $900 \mathrm{~kb} / \mathrm{cM}$. However, such recombination rate estimates may vary greatly within and between chromosomes and reliable estimates of recombination rates per physical distance are impossible to obtain in the absence of physical maps (eg Beye et al, 1999). 
Currently, we have no information hinting at the function of api. Further characterization of api will therefore provide basic information on the molecular control mechanisms governing the expression of this wing polymorphism. With the identification of api, we may also gain insights into the molecular mechanisms underlying an environmentally sensitive expression of alternative phenotypes. The wing polyphenism in parthenogenetic pea aphids generates winged and wingless females that closely resemble the different phenotypes observed in the male wing polymorphism. Therefore, polymorphic and polyphenic expressions of these alternative phenotypes share some of their underlying developmental processes; yet, it is unclear whether and how api is involved in the divergence of environmentally induced alternatives. Initial results indicate that allelic variation at api may correlate with genetic differences in the propensity to produce winged females, indicating genetic linkage of factors controlling the wing polyphenism and wing polymorphism (Braendle et al, in press). Characterizing api and testing its functional relevance during the development of alternative phenotypes in the wing polyphenism will further address these questions. This, in turn, may shed light on the molecular genetic basis of adaptive phenotypic plasticity and the evolutionary transitions between polyphenism and polymorphism.

\section{Acknowledgements}

We thank Andy DeWan, Greg Hunt and Alejandro Schäffer for advice on the mapping protocol, David Hawthorne for providing primer data and advice on AFLP analysis and Mauro Mandrioli for comments on an earlier version of the paper. CB acknowledges financial support by the Boehringer Ingelheim Fonds, the Roche Research Foundation and the Janggen-Poehn-Stiftung.

\section{References}

Beye M, Hunt GJ, Page RE, Fondrk MK, Grohmann L, Moritz RFA (1999). Unusually high recombination rate detected in the sex locus region of the honey bee (Apis mellifera). Genetics 153: 1701-1708.

Birkle LM, Douglas AE (1999). Low genetic diversity among pea aphid (Acyrthosiphon pisum) biotypes of different plant affiliation. Heredity 82: 605-612.

Bizzaro D, Mandrioli M, Zanotti M, Giusti M, Manicardi GC (2000). Chromosome analysis and molecular characterization of highly repeated DNAs in the aphid Acyrthosiphon pisum (Aphididae, Hemiptera). Genetica 108: 197-202.

Blackman RL (1987). Reproduction, cytogenetics and development. In: Minks AK, Harrewijn P (eds) Aphids: Their Biology, Natural Enemies \& Control. Elsevier: Amsterdam. Vol 2A, pp 163-195.

Boulding EG (1998). Molecular evidence against phylogenetically distinct host races of the pea aphid (Acyrthosiphon pisum). Genome 41: 769-775.

Braendle C, Friebe I, Caillaud CM, Stern DL. Genetic variation for an aphid wing polyphenism is genetically linked to a naturally occurring wing polymorphism. Proc R Soc London $B$ (in press).

Caillaud MC, Boutin M, Braendle C, Simon J-C (2002). A sexlinked locus controls wing polymorphism in males of the pea aphid, Acyrthosiphon pisum (Harris). Heredity 89: 346-352.
Dalmasso A, Bournoville R (1983). Isozymes d'une population d'Acyrthosiphon pisum Harris (Hom., Aphididae). Agronomie (Paris) 3: 891-896.

Evans JD, Wheeler DE (1999). Differential gene expression between developing queens and workers in the honey bee, Apis mellifera. Proc Natl Acad Sci USA 96: 5575-5580.

Finston TL, Hebert PDN, Foottit RB (1995). Genome size variation in aphids. Insect Biochem Mol Biol 25: 189-196.

Fukatsu T (1999). Acetone preservation: a practical technique for molecular analysis. Mol Ecol 8: 1935-1945.

Harrison RG (1980). Dispersal polymorphisms in insects. Annu Rev Ecol Syst 11: 95-118.

Hawthorne DJ (2001). AFLP-based genetic linkage map of the Colorado Potato Beetle Leptinotarsa decemlineata: sex chromosomes and a pyrethroid-resistance candidate gene. Genetics 158: $695-700$.

Hawthorne DJ, Via S (2001). Genetic linkage of ecological specialization and reproductive isolation in pea aphids. Nature 412: 904-907.

Kawada K (1987). Forms and morphs of aphids. In: Minks AK, Harrewijn P (eds) Aphids, Their Biology, Natural Enemies and Control. Elsevier: Amsterdam. Vol 2A, pp 255-266.

Kosambi DD (1944). The estimation of map distances from recombination values. Ann Eugenics 12: 172-175.

Kring JB (1977). Structure of the eyes of the pea aphid, Acyrthosiphon pisum. Ann Entomol Soc Am 70: 855-860.

Lander ES, Green P, Abrahamson J, Barlow A, Daly MJ, Lincoln SE et al (1987). MAPMAKER: an interactive computer package for constructing primary genetic linkage maps of experimental and natural populations. Genomics 1: 174-181.

Lees AD (1966). The control of polymorphism in aphids. Adv Insect Physiol 3: 207-277.

Lincoln SE, Lander ES (1992). Systematic detection of errors in genetic linkage data. Genomics 14: 604-610.

Mandrioli M, Bizzaro D, Giusti M, Manicardi GC, Bianchi U (1999). The role of rDNA genes in X chromosome association in the aphid Acyrthosiphon pisum. Genome 42: 381-386.

Miura T, Braendle C, Shingleton A, Sisk G, Kambhampati S, Stern DL (2003). A comparison of parthenogenetic and sexual embryogenesis of the pea aphid Acyrthosiphon pisum (Hemiptera: Aphidoidea). J Exp Zool (Mol Dev Evol) 295B: 59-81.

Miura T, Kamikouchi A, Sawata M, Takeuchi H, Natori S, Kubo $\mathrm{T}$ et al (1999). Soldier caste-specific gene expression in the mandibular glands of Hodotermopsis japonica (Isoptera: Termopsidae). Proc Natl Acad Sci USA 96: 13874-13879.

Miyazaki M (1987). Forms and morphs of aphids. In: Minks AK, Harrewijn P (eds) Aphids, Their Biology, Natural Enemies and Control. Elsevier: Amsterdam. Vol 2A, pp 163-195.

Moran NA (1992). The evolutionary maintenance of alternative phenotypes. Am Nat 139: 971-989.

Parsons YM, Shaw KL (2002). Mapping unexplored genomes: a genetic linkage map of the Hawaiian cricket Laupala. Genetics 162: $1275-1282$.

Roff DA (1986). The evolution of wing dimorphism in insects Evolution 40: 1009-1020.

Roff DA (1996). The evolution of threshold traits. Q Rev Biol 71: 3-35.

Roff DA, Fairbairn DJ (1991). Wing dimorphisms and the evolution of migratory polymorphisms among the Insecta. Am Zool 31: 243-251.

Simon JP, Parent MA, Auclair JL (1982). Isozyme analysis of biotypes and field populations of the pea aphid. Entomol Exp Appl 32: 186-192.

Smith MAH, MacKay PA (1989). Genetic variation in male alary dimorphism in populations of the pea aphid, Acyrthosiphon pisum. Entomol Exp Appl 51: 125-132.

Sun R-Y, Robinson AG (1966). Chromosome studies on 50 species of aphids. Can J Zool 44: 649-653.

Via S (1992). Inducing the sexual forms and hatching eggs of pea aphids. Entomol Exp Appl 65: 119-127. 
442

Voorrips RE (2002). MapChart: software for the graphical presentation of linkage maps and QTLs. J Hered 93: 77-78.

Vos P, Hogers R, Bleeker M, Reijans M, Van de Lee T, Hornes M (1995). AFLP: a new technique for DNA fingerprinting. Nucleic Acids Res 23: 4407-4414.

West-Eberhard MJ (1989). Phenotypic plasticity and the origins of diversity. Annu Rev Ecol Syst 20: 249-278.

Zar JH (1999). Biostatistical Analysis. Prentice-Hall: Englewood Cliffs, NJ.
Zera AJ, Denno RF (1997). Physiology and ecology of dispersal polymorphism in insects. Annu Rev Entomol 42: 207-231.

Zera AJ, Harshman LG (2001). The physiology of life history trade-offs in animals. Annu Rev Ecol Syst 32: 95-126.

\section{Appendix A1}

The pattern of male morph production in the $\mathrm{F}_{2}$ hybrid clones used for mapping is shown in Table A1.

Table A1 Pattern of male morph production in the $78 \mathrm{~F}_{2}$ hybrid clones used for mappinga

\begin{tabular}{|c|c|c|c|c|c|c|c|}
\hline$F_{2}$ cross & No. & Winged males & Wingless males & $F_{2}$ cross & No. & Winged males & Wingless males \\
\hline 1 & 4 & 3 & 6 & 3 & 73 & 13 & 9 \\
\hline 1 & 21 & 1 & 1 & 3 & 76 & 7 & 6 \\
\hline 1 & 23 & 13 & 9 & 3 & 79 & 19 & 15 \\
\hline 1 & 32 & 5 & 9 & 3 & 82 & 22 & 15 \\
\hline 1 & 36 & 12 & 6 & 3 & 84 & 13 & 16 \\
\hline 2 & 4 & 16 & 19 & 3 & 93 & 7 & 4 \\
\hline 2 & 16 & 4 & 5 & 3 & 94 & 1 & 4 \\
\hline 2 & 20 & 4 & 9 & 3 & 97 & 12 & 22 \\
\hline 2 & 26 & 14 & 13 & 3 & 98 & 17 & 23 \\
\hline 2 & 31 & 30 & 26 & 3 & 104 & 10 & 14 \\
\hline 2 & 35 & 4 & 7 & 3 & 106 & 38 & 47 \\
\hline 2 & 61 & 7 & 8 & 3 & 107 & 11 & 11 \\
\hline 2 & 77 & 28 & 28 & 3 & 109 & 3 & 3 \\
\hline 2 & 78 & 5 & 22 & 3 & 113 & 13 & 20 \\
\hline 2 & 93 & 5 & 11 & 3 & 117 & 12 & 15 \\
\hline 2 & 95 & 11 & 11 & 3 & 125 & 9 & 12 \\
\hline 2 & 102 & 8 & 12 & 3 & 132 & 58 & 48 \\
\hline 2 & 104 & 11 & 15 & 3 & 136 & 21 & 13 \\
\hline 2 & 106 & 15 & 13 & 3 & 137 & 12 & 25 \\
\hline 2 & 120 & 12 & 12 & 3 & 139 & 5 & 10 \\
\hline 2 & 122 & 4 & 4 & 3 & 143 & 4 & 12 \\
\hline 2 & 128 & 24 & 12 & 3 & 157 & 9 & 11 \\
\hline 2 & 155 & 10 & 10 & 3 & 159 & 20 & 24 \\
\hline 2 & 160 & 10 & 12 & 3 & 168 & 24 & 13 \\
\hline 3 & 8 & 27 & 37 & 3 & 176 & 16 & 15 \\
\hline 3 & 22 & 6 & 4 & 3 & 189 & 29 & 20 \\
\hline 3 & 24 & 3 & 1 & 3 & 191 & 29 & 27 \\
\hline 3 & 25 & 26 & 34 & 3 & 192 & 2 & 6 \\
\hline 3 & 26 & 4 & 5 & 3 & 193 & 5 & 5 \\
\hline 3 & 32 & 9 & 6 & 3 & 196 & 8 & 19 \\
\hline 3 & 35 & 5 & 5 & 3 & 204 & 12 & 10 \\
\hline 3 & 38 & 3 & 2 & 3 & 210 & 11 & 10 \\
\hline 3 & 49 & 13 & 18 & 4 & 71 & 1 & 1 \\
\hline 3 & 56 & 18 & 14 & 4 & 79 & 8 & 7 \\
\hline 3 & 60 & 6 & 4 & 4 & 90 & 19 & 19 \\
\hline 3 & 61 & 8 & 16 & 4 & 128 & 22 & 15 \\
\hline 3 & 63 & 10 & 14 & 4 & 140 & 4 & 2 \\
\hline 3 & 67 & 2 & 5 & 4 & 174 & 27 & 34 \\
\hline 3 & 70 & 18 & 19 & & & & \\
\hline
\end{tabular}

${ }^{a}$ A heterogeneity $\chi^{2}$ test (Zar, 1999) shows that, overall, the proportions of winged and wingless males are not different $(P>0.05)$. 